\title{
STOCHASTIC STABILITY OF MEASURES IN GRADIENT SYSTEMS
}

\author{
WEN HUANG, MIN JI, ZHENXIN LIU, AND YINGFEI YI
}

\begin{abstract}
Stochastic stability of a compact invariant set of a finite dimensional, dissipative system is studied in our recent work "Concentration and limit behaviors of stationary measures" ([20]) for general white noise perturbations. In particular, it is shown under some Lyapunov conditions that the global attractor of the systems is always stable under general noise perturbations and any strong local attractor in it can be stabilized by a particular family of noise perturbations. Nevertheless, not much is known about the stochastic stability of an invariant measure in such a system. In this paper, we will study the issue of stochastic stability of invariant measures with respect to a finite dimensional, dissipative gradient system with potential function $f$. As we will show, a special property of such a system is that it is the set of equilibria which is stable under general noise perturbations and the set $S_{f}$ of global minimal points of $f$ which is stable under additive noise perturbations. For stochastic stability of invariant measures in such a system, we will characterize two cases of $f$, one corresponding to the case of finite $S_{f}$ and the other one corresponding to the case when $S_{f}$ is of positive Lebesgue measure, such that either some combined Dirac measures or the normalized Lebesgue measure on $S_{f}$ is stable under additive noise perturbations. However, we will show by constructing an example that such measure stability can fail even in the simplest situation, i.e., in 1-dimension there exists a potential function $f$ such that $S_{f}$ consists of merely two points but no invariant measure of the corresponding gradient system is stable under additive noise perturbations. Crucial roles played by multiplicative and additive noise perturbations to the measure stability of a gradient system will also be discussed. In particular, the nature of instabilities of the normalized Lebesgue measure on $S_{f}$ under multiplicative noise perturbations will be exhibited by an example.
\end{abstract}

\section{INTRODUCTION}

Consider a system of ordinary differential equations

$$
\dot{x}=V(x), \quad x \in \mathbb{R}^{n},
$$

where $V=\left(V^{i}\right) \in C\left(\mathbb{R}^{n}, \mathbb{R}^{n}\right)$. Adding multiplicative including additive white noise $G(x) \dot{W}$ to (1.3), we obtain the following Itô stochastic differential equations

$$
\mathrm{d} x=V(x) \mathrm{d} t+G(x) \mathrm{d} W, \quad x \in \mathbb{R}^{n},
$$

2000 Mathematics Subject Classification. Primary 34Dxx, 37C75, 37C40; Secondary 60H10, 35Q84.

Key words and phrases. Fokker-Planck equation, Gradient systems, Gibbs measure, Limit measure, Stochastic stability, White noise perturbation.

Corresponding author: Y. Yi (yingfei@ualberta.ca,yi@math.gatech.edu).

The first author was partially supported by NSFC grants 11225105, 11371339. The second author was partially supported by NSFC Innovation grant 10421101 and NSFC grant 11571344. The third author was partially supported by NSFC grants 11271151, 11522104, and the startup and Xinghai Youqing funds from Dalian University of Technology. The fourth author was partially supported by NSF grant DMS1109201, NSERC discovery grant 1257749, a faculty development grant from University of Alberta, and a Scholarship from Jilin University. The work was partially done while the authors were visiting each other's Institutions. 
where $W$ is a standard $m$-dimensional Brownian motion for some integer $m \geq n$ and $G=$ $\left(g^{i j}\right)_{n \times m}$ is a matrix-valued function on $\mathbb{R}^{n}$.

Regarded as a physical model, system (1.1) is often subject to white noise perturbations either from its surrounding environment or from intrinsic uncertainties of a coupling system due to high complexity, large degree of freedom, lack of full knowledge of mechanisms, the need for organizing a large amount of data, etc. Suppose that (1.1) generates a local flow on $\mathbb{R}^{n}$. Analyzing the impact of noise perturbations on the dynamics of the system is a fundamental issue with respect to both modeling and dynamics. The study of this fundamental issue from a distribution point of view naturally gives rise to the analysis of limit behaviors of stationary measures of the Fokker-Planck equations associated with (1.2) as $G \rightarrow 0$ under an appropriate topology.

More precisely, consider noise coefficient matrices lying in the class

$$
\tilde{\mathcal{G}}=\left\{G=\left(g^{i j}\right): \operatorname{Rank}(G) \equiv n, g^{i j} \in W_{l o c}^{1,2 p}\left(\mathbb{R}^{n}\right), i=1,2, \cdots, n, j=1,2, \cdots, m\right\}
$$

for some fixed $p>n$. The class $\tilde{\mathcal{G}}$ gives rise to the following class of diffusion matrices:

$$
\tilde{\mathcal{A}}=\left\{A=\left(a^{i j}\right) \in W_{l o c}^{1, p}\left(\mathbb{R}^{n}, G L(n, \mathbb{R})\right): A=\frac{G G^{\top}}{2} \text { for some } G \in \tilde{\mathcal{G}}\right\} .
$$

For each $A=\frac{G G^{\top}}{2}=\left(a^{i j}\right) \in \tilde{\mathcal{A}}$, the stationary process generated by (1.2) is described by its corresponding stationary measures $\left\{\mu_{A}\right\}$ which are measure-valued solutions of the stationary Fokker-Planck equation associated with (1.2) (see Section 2.1 for details).

Let $\tilde{\mathcal{A}}$ be equipped with the uniform topology of $C\left(\mathbb{R}^{n}\right)$ and consider an admissible null family $\mathcal{A}=\left\{A_{\alpha}\right\} \subset \tilde{\mathcal{A}}$, i.e., $\mathcal{A}$ is a directed net with $A_{\alpha} \rightarrow 0$, and the Fokker-Planck equation corresponding to each $A_{\alpha} \in \mathcal{A}$ admits a stationary measure. Stability of dynamics of (1.1) under the noise family $\mathcal{A}$ can be characterized by the behaviors of $\mathcal{A}$-limit measures, i.e., sequential limit points of all stationary measures $\left\{\mu_{A_{\alpha}}\right\}$ of the Fokker-Planck equations corresponding to $\mathcal{A}=\left\{A_{\alpha}\right\}$, as $A_{\alpha} \rightarrow 0$, in the space $M\left(\mathbb{R}^{n}\right)$ of Borel probability measures on $\mathbb{R}^{n}$ endowed with the weak*-topology. We recall from [20] that a compact invariant set $\Omega$ of (1.1) is said to be $\mathcal{A}$-stable if for any $\epsilon>0$ and any open neighborhood $W$ of $\Omega$ there exists a $\delta>0$ such that $\mu_{A_{\alpha}}\left(\mathbb{R}^{n} \backslash W\right)<\epsilon$ whenever $\left|A_{\alpha}\right|<\delta$. An invariant measure $\mu$ of (1.1) is said to be $\mathcal{A}$-stable if any sequence of $\left\{\mu_{A_{\alpha}}\right\}$ converges to $\mu$ in $M\left(\mathbb{R}^{n}\right)$ as $A_{\alpha} \rightarrow 0$, i.e., $\mu$ is the only $\mathcal{A}$-limit measure.

$\mathcal{A}$-stability of a compact invariant set of (1.1) has been extensively investigated in [20]. In particular, it is shown in [20] that if (1.1) admits a Lyapunov function whose second derivatives are bounded, then its global attractor is $\mathcal{A}$-stable with respect to any null family $\mathcal{A}$, and moreover, if the global attractor contains a strong local attractor then there is an admissible null family $\mathcal{A}$ such that the local attractor is $\mathcal{A}$-stable. To the contrary, if the global attractor contains a strong local repeller, then there is an admissible null family $\mathcal{A}$ such that the local repeller is strongly $\mathcal{A}$-unstable, i.e., no $\mathcal{A}$-limit measure can be concentrated on the repeller. Moreover, if the repeller is a so-called strongly repelling equilibrium, then it is strongly $\mathcal{A}$-unstable with respect to any so-called normal null family $\mathcal{A}$.

In contrast to the case of compact invariant sets, not much is known about the $\mathcal{A}$-stability of invariant measures of (1.1). Of course, if an $\mathcal{A}$-stable compact invariant set is uniquely ergodic, then it is clear that the unique invariant measure is also $\mathcal{A}$-stable. In general, 
stochastic stability of a non-ergodic invariant measure is much harder to be characterized. We mention some well-known studies in this regard for flows on a 2-torus ([23]), flows on a circle $([27])$, flows whose $\omega$-limit sets consist of a finite number of fixed points and periodic orbits ([28]), and flows on a compact manifold admitting SRB measures ([24], see also [11, 29] for the case of random perturbations of maps on a compact manifold).

In this paper, we pay particular attention to the stochastic stability of compact invariant sets and invariant measures of a gradient system

$$
\dot{x}=-\nabla f(x), \quad x \in \mathbb{R}^{n},
$$

where $f \in C^{2}\left(\mathbb{R}^{n}\right)$.

As to be seen in this paper, not only does the stochastic stability of compact invariant sets of (1.3) has very special natures, but also the stochastic stability of invariant measures of (1.3) can be characterized in various situations. Besides analyzing the impact of noise perturbations on a gradient system, the study of stochastic stability of invariant measures in a gradient system is closely related to the problem of ergodicity when taking the thermodynamic limit in a huge particle system $([1])$ and the problem of noise stabilizing a multi-stable gradient system $([10])$.

It is well-known that if the gradient system (1.3) admits a weak Lyapunov function then it always generates a positive semiflow in $\mathbb{R}^{n}$. Another special property of a gradient system is that when it is dissipative, its global attractor is typically simple by consisting of equilibria together with connecting orbits among them. However, it follows from general results of [20] that noise perturbations can remove all the connecting orbits among the equilibria in the global attractor of a dissipative gradient system. More precisely, if (1.3) admits a $C^{2}$ Lyapunov function whose second derivative is bounded, then a) the set $E$ of critical points of $f$ is $\mathcal{A}$-stable with respect to any null family $\mathcal{A}$; b) any finite set $\mathcal{J}_{0}$ (resp. $\mathcal{R}_{0}$ ) of isolated local minimal (maximal) points of $f$ is $\mathcal{A}$-stable (resp. strongly $\mathcal{A}$-unstable) with respect to a particular null family $\mathcal{A}$; c) any simple local maximal point of $f$ is strongly $\mathcal{A}$-unstable with respect to any normal null family $\mathcal{A}$ (see Theorem 2.1 for details). When either the set $E$ or $\mathcal{J}_{0}$ is a singleton, the $\mathcal{A}$-stability of the corresponding Dirac measure obviously follows from that of the $E$ or $\mathcal{J}_{0}$ with respect to all or a particular null family $\mathcal{A}$.

However, stochastic stability of (1.3) has very special natures under the additive white noise perturbation $\sqrt{2 \epsilon} \dot{W}$ to (1.3), where $\epsilon>0$ is a small parameter. Under the condition that

H) there are positive constants $R$ and $\beta$ such that $f(x) \geq \beta \log |x|$ for all $|x| \geq R$, the Fokker-Planck equation corresponding to

$$
\mathrm{d} x=-\nabla f(x) \mathrm{d} t+\sqrt{2 \epsilon} \mathrm{d} W, \quad x \in \mathbb{R}^{n}
$$

for each $\epsilon>0$ admits a stationary measure $\mu_{\epsilon}$, called Gibbs measure, with density

$$
u^{\epsilon}(x)=k_{\epsilon} \mathrm{e}^{-\frac{f(x)}{\epsilon}}, \quad x \in \mathbb{R}^{n},
$$

called Gibbs state, where

$$
k_{\epsilon}=\frac{1}{\int_{\mathbb{R}^{n}} \mathrm{e}^{-\frac{f(x)}{\epsilon}} \mathrm{d} x} .
$$


Limit behaviors of (continuum) Gibbs measures have been explicitly investigated in many situations (see, e.g., $[3,21]$ ). These limit behaviors lead to various $\mathcal{A}_{0}$-stability results of (1.3), where $\mathcal{A}_{0}$ denote the family of diffusion matrices $\{\epsilon I\}$ corresponding to the additive noise perturbations. More precisely, it follows from the limit characterizations of Gibbs measures in [21] that if the condition $\mathbf{H}$ ) holds, then the set $S_{f}$ of all global minimal points of $f$ is $\mathcal{A}_{0^{-}}$ stable (see Theorem $2.2 \mathrm{a}$ )). This result actually reveals a special stochastic stability feature of (1.3) under additive noise perturbations because it implies that any compact invariant set of (1.3) consisting of relative minimal points of $f$ as well as any invariant measure on the set will not be $\mathcal{A}_{0}$-stable though by Theorem 2.1 they can be $\mathcal{A}$-stable with respect to some null family $\mathcal{A}$ of multiplicative noise perturbations.

If $S_{f}$ is a singleton, then the stability of $S_{f}$ under additive noise perturbation implies that of its Dirac measure. In the case that $S_{f}$ is not a singleton, $\mathcal{A}_{0}$-stability of a broader class of non-ergodic invariant measures on $S_{f}$ can also be studied using existing convergence results of Gibbs measures $([3,21])$. It turns out that such measure stability depends on the nature of absolute minimal points of $f$. For instance, there are $\mathcal{A}_{0}$-stable invariant measures in the following two special cases:

C1) $f \in C^{2}\left(\mathbb{R}^{n}\right), S_{f}=\left\{x_{1}, x_{2}, \cdots, x_{m}\right\}$ for some $m \in \mathbb{N}$, and near each $x_{i}, i=1,2, \cdots, m$, $f$ has the form

$$
f(x)=P_{i}\left(x-x_{i}\right)+o\left(\left|x-x_{i}\right|^{2 k_{i}}\right), \quad x \in B_{i}:=B_{\delta}\left(x_{i}\right),
$$

where $P_{i}$ is a homogeneous polynomial of order $2 k_{i}$ for some natural number $k_{i}$ and $B_{\delta}\left(x_{i}\right)$ is the open $\delta$-ball centered at $x_{i}$ for some $\delta>0$.

C2) $S_{f}$ admits positive Lebesgue measure.

More precisely, in the case $\mathbf{C} 1$ ), the $\mathcal{A}_{0}$-stable measure has the form $\sum_{i=1}^{m} c_{i} \delta_{x_{i}}$, where, for each $i=1, \cdots, m, \delta_{x_{i}}$ denotes the Dirac measure at $x_{i}$ and $c_{i}$ depends on $P_{i}$, and in the case C2), the $\mathcal{A}_{0}$-stable measure is precisely the normalized Lebesgue measure $\mu_{f}$ on $S_{f}$ (see Theorem $2.2 \mathrm{~b}), \mathrm{c})$ ). We note that (1.5) holds automatically when $n=1$. In general, when $S_{f}$ consists of a finite number of points and each point is an isolated minimum point of $f$, it is only known that the leading term of the Taylor expansion near each of these points must be of even order.

Stochastic stability of an invariant measure is a much more restrictive property than that of a compact invariant set. One of the main goals of this paper is to show, even in the simplest situation, that if an $\mathcal{A}$-stable compact invariant set of a finite dimensional flow is non-uniquely ergodic, then it can occur that none of its invariant measures is $\mathcal{A}$-stable. We will demonstrate such an instability phenomenon by giving a 1-dimensional example under additive noise perturbation such that the condition in C1) fails in the simplest case $m=2$ (Note that if $m=1$ then the Dirac measure on $S_{f}$ is always $\mathcal{A}_{0}$-stable). Main features of this example are summarized as follows.

Theorem 1. (Measure instability under additive noise) In the case $n=1$ there exists a potential function $f \in C^{2}(\mathbb{R})$ for which the following properties hold under additive noise perturbation $\sqrt{2 \epsilon} \dot{W}$. 
a) $S_{f}$ consists of two points $x_{1}, x_{2}$ but neither is an isolated critical point of $f$.

b) The corresponding Fokker-Planck equation admits no stationary measures other than the Gibbs measures $\left\{\mu_{\epsilon}\right\}$.

c) There are two sequences $\epsilon_{k}^{1}, \epsilon_{k}^{2} \rightarrow 0$ such that $\mu_{\epsilon_{k}^{i}} \rightarrow \lambda_{i} \delta_{x_{1}}+\left(1-\lambda_{i}\right) \delta_{x_{2}}$ as $k \rightarrow \infty$, for $i=1,2$ respectively, where $\lambda_{1}, \lambda_{2} \in(0,1)$ are two distinct constants. Consequently, no invariant measure on $S_{f}$ is $\mathcal{A}_{0}$-stable.

Theorem 1 is also a counter example when the condition in C2) fails. When the condition in C2) holds, we note that the stability of the normalized Lebesgue measure on $S_{f}$ can be however destroyed by a multiplicative noise perturbation, indicating an impact of multiplicative noise perturbations on the instability of non-singular measures. We will give an example to demonstrate such an instability phenomenon. Main features of this example are summarized as follows.

Theorem 2. (Measure instability under multiplicative noise) In the case $n=1$ there exists a potential function $f \in C^{2}(\mathbb{R})$ satisfying the condition in $\mathbf{C 2}$ ) and infinitely many null families $\mathcal{A}$ satisfying the following properties:

a) $S_{f}$ is $\mathcal{A}$-stable.

b) Any $\mathcal{A}$-limit measure differs from the normalized Lebesgue measure $\mu_{f}$ on $S_{f}$, i.e., $\mu_{f}$ is $\mathcal{A}$-unstable.

This paper is organized as follows. In Section 2, we review some basic results on stationary measures and stochastic stability, and give some results on the stochastic stability of compact invariant sets of (1.3) under both multiplicative and additive noise perturbations. In Section 3, we construct a 1-dimensional example having the features described in Theorem 1 whose Gibbs measures are not convergent as $\epsilon \rightarrow 0$. In Section 4, we construct an example of gradient system having the features described in Theorem 2.

Throughout the rest of the paper, for simplicity, we will use the same symbol $|\cdot|$ to denote the absolute value of a number, the norm of a vector or a matrix, and the Lebesgue measure of a set.

\section{Stability Under additive V.S. multiplicative NOISES}

In this section, we will discuss stochastic stability of invariant sets and invariant measures of (1.3) under both additive and multiplicative noise perturbations. Through the rest of the section, for simplicity, we will use short notions $\partial_{i}=\frac{\partial}{\partial x_{i}}, \partial_{i j}^{2}=\frac{\partial^{2}}{\partial x_{i} \partial x_{j}}$, and we also adopt the usual summation convention on $i, j=1,2, \cdots, n$ whenever applicable.

2.1. Lyapunov functions and stationary measures. Adding multiplicative white noise $G(x) \dot{W}$ to (1.3), where $W$ is a standard $m$-dimensional Brownian motion for some integer $m \geq n$ and $G=\left(g^{i j}\right)_{n \times m}$ is a matrix-valued function on $\mathbb{R}^{n}$, we obtain the following Itô stochastic differential equations

$$
\mathrm{d} x=-\nabla f(x) \mathrm{d} t+G(x) \mathrm{d} W, \quad x \in \mathbb{R}^{n} .
$$

Let $A=\frac{G G^{\top}}{2}=\left(a^{i j}\right)$ and consider the adjoint Fokker-Planck operator

$$
\mathcal{L}_{A}=a^{i j} \partial_{i j}^{2}-\partial_{i} f \partial_{i}
$$


corresponding to $A$. A stationary measure corresponding to $\mathcal{L}_{A}$ is a stationary measure of the Fokker-Planck equation associated with (2.1), i.e., a Borel probability measure $\mu$ satisfying

$$
\int_{\mathbb{R}^{n}} \mathcal{L}_{A} F(x) \mathrm{d} \mu(x)=0, \quad \forall F \in C_{0}^{\infty}\left(\mathbb{R}^{n}\right) .
$$

A weak stationary solution corresponding to $\mathcal{L}_{A}$ is a weak stationary solution of the FokkerPlanck equation associated with (2.1), i.e., a continuous function $u$ on $\mathbb{R}^{n}$ satisfying

$$
\left\{\begin{array}{l}
\int_{\mathcal{U}} \mathcal{L}_{A} F(x) u(x) \mathrm{d} x=0, \quad \forall F \in C_{0}^{\infty}(\mathcal{U}), \\
u(x) \geq 0, \quad \int_{\mathbb{R}^{n}} u(x) \mathrm{d} x=1 .
\end{array}\right.
$$

Corresponding to $\mathcal{L}_{A}$, if $u$ is a weak stationary solution, then it is clear that $\mu$ with $\mathrm{d} \mu(x)=$ $u(x) \mathrm{d} x$ is a stationary measure. Conversely, by the regularity theorem in [4], if $A \in \tilde{\mathcal{A}}$ then a stationary measure $\mu$ corresponding to $\mathcal{L}_{A}$ must be regular in the sense that $\mathrm{d} \mu(x)=u(x) \mathrm{d} x$ for a density function $u \in W_{l o c}^{1, p}\left(\mathbb{R}^{n}\right)$ which is a weak stationary solution corresponding to $\mathcal{L}_{A}$. We note that if $a^{i j} \in C_{l o c}^{2, \alpha}\left(\mathbb{R}^{n}\right), i, j=1, \cdots, n$, and $f \in C_{l o c}^{2, \alpha}\left(\mathbb{R}^{n}\right)$, for some $\alpha \in(0,1)$, then it follows from the standard Schauder theory that a weak stationary solution corresponding to $\mathcal{L}_{A}$ becomes a classical solution of the stationary Fokker-Planck equation

$$
\left\{\begin{array}{l}
\partial_{i j}^{2}\left(a^{i j} u\right)+\partial_{i}\left(\partial_{i} f u\right)=0, \\
u(x) \geq 0, \quad \int_{\mathbb{R}^{n}} u(x) \mathrm{d} x=1
\end{array}\right.
$$

associated with (2.1).

We recall from $[16,17]$ that a non-negative continuous function $U$ on $\mathbb{R}^{n}$ is a compact function if $U(x)<\rho_{M}, x \in \mathbb{R}^{n}$, and $\lim _{x \rightarrow \infty} U(x)=\rho_{M}$, where $\rho_{M}=\sup _{x \in \mathbb{R}^{n}} U(x) \leq \infty$. It is easy to see that a non-negative function $U$ is an unbounded compact function if and only if $\lim _{x \rightarrow \infty} U(x)=+\infty$. A $C^{2}$ compact function $U$ on $\mathbb{R}^{n}$ is called a Lyapunov function with respect to (2.1) or $\mathcal{L}_{A}$ if there is a constant $\rho_{m}<\rho_{M}$ and a constant $\gamma>0$, called Lyapunov constant of $U$, such that

$$
\mathcal{L}_{A} U(x) \leq-\gamma, \quad \text { whenever } U(x)>\rho_{m} .
$$

Let $\mathcal{A}=\left\{A_{\alpha}\right\}$ be a null family. Recall from [19] that a compact function $U \in C^{2}\left(\mathbb{R}^{n}\right)$ is a uniform Lyapunov function with respect to the family $\left\{\mathcal{L}_{A_{\alpha}}\right\}$ if (2.5) holds for constants $\rho_{m}, \gamma$ that are independent of $A_{\alpha} \in \mathcal{A}$.

The concept of Lyapunov function above generalizes the usual one for a deterministic system. Recall that a $C^{1}$ compact function $U$ on $\mathbb{R}^{n}$ is called a Lyapunov function of (1.3) if there exist constants $0 \leq \rho_{m}<\rho_{M}, \gamma>0$ such that

$$
-\nabla f(x) \cdot \nabla U(x) \leq-\gamma, \quad \text { whenever } U(x)>\rho_{m} .
$$

If (2.6) holds with $\gamma=0$ and some $\rho_{m}<\rho_{M}$, then $U$ is called a weak Lyapunov function of (1.3). A weak Lyapunov function $U$ is called an entire weak Lyapunov function of (1.3) if $\rho_{m}=0$ in the above. In fact, the notions of compact, Lyapunov, weak Lyapounov, and entire weak Lyapunov functions can be introduced on any connected open subset of $\mathbb{R}^{n}$ instead of the entire $\mathbb{R}^{n}$ (see [17] for details). In particular, for a connected open set $\mathcal{U} \subset \mathbb{R}^{n}$, a compact function $U \in C^{1}(\mathcal{U}, \mathbb{R})$ is called an entire weak Lyapunov function of (1.3) in $\mathcal{U}$ if

$$
-\nabla f(x) \cdot \nabla U(x) \leq 0, \quad x \in \mathcal{U} .
$$


Remark 2.1. Let $\varphi^{t}$ denote the local flow generated from (1.3). According to the general theory of dissipative systems (see e.g., [18, Proposition 6.3]), if (1.3) admits a weak Lyapunov function, then $\varphi^{t}$ becomes a semiflow on $\mathbb{R}^{n}$, and moreover, if (1.3) admits a Lyapunov function, then $\varphi^{t}$ must be dissipative and hence admit a global attractor. Let $E$ denote the set of equilibria of (1.3). It is well-known that the global attractor of $\varphi^{t}$, if exists, equals the unstable set of $E$, which, in typical situations, consists of equilibria of (1.3) along with their connecting orbits. The global attractor of $\varphi^{t}$ is actually invariant with respect to $\varphi^{t}$, i.e., $\varphi^{t}$ can be extended to a flow on it.

We note that for any null family $\mathcal{A}=\left\{A_{\alpha}\right\}$ a uniform Lyapunov function with respect to $\left\{\mathcal{L}_{A_{\alpha}}\right\}$ is necessarily a Lyapunov function of (1.3). Conversely, if (1.3) admits a $C^{2}$ Lyapunov function whose second derivative is bounded, then with respect to any null family $\mathcal{A}=\left\{A_{\alpha}\right\}$, this Lyapunov function is clearly a uniform Lyapunov function with respect to the family $\left\{\mathcal{L}_{A_{\alpha}}\right\}$ as $\left|A_{\alpha}\right| \ll 1$. In the case $\mathcal{A}=\{\epsilon I\}$, where $\epsilon>0$ is a small parameter, it is easy to see that if $U$ is a Lyapunov function of (1.3), then it is a uniform Lyapunov function with respect to the family $\left\{\mathcal{L}_{\epsilon I}\right\}$ if either $\Delta U$ is bounded on $\mathbb{R}^{n}$ or $U$ is sub-harmonic and a Lyapunov function with respect to $\mathcal{L}_{I}$.

For a given null family, the following result summarizes important roles played by a uniform Lyapunov function to the corresponding stationary measures.

Lemma 2.1. Consider a null family $\mathcal{A}=\left\{A_{\alpha}\right\}$ and assume that there is a uniform Lyapunov function with respect to the family $\left\{\mathcal{L}_{A_{\alpha}}\right\}$. Then the following holds.

a) (Admissibility) There is a stationary measure corresponding to each $\mathcal{L}_{A_{\alpha}}$ which is regular with density function lying in the space $W_{\text {loc }}^{1, p}\left(\mathbb{R}^{n}\right)$. Consequently, $\mathcal{A}$ is admissible.

b) (Tightness) As $A_{\alpha} \rightarrow 0$, the family $\left\{\mu_{A_{\alpha}}\right\}$ of all stationary measures corresponding to $\left\{\mathcal{L}_{A_{\alpha}}\right\}$ is relatively sequentially compact in $M\left(\mathbb{R}^{n}\right)$.

c) (Concentration) Any limit measure of $\left\{\mu_{A_{\alpha}}\right\}$, as $A_{\alpha} \rightarrow 0$, is an invariant measure of $\varphi^{t}$ supported on the global attractor of $\varphi^{t}$.

In the above lemma, a) follows from general existence results contained in [7, 17], and b), c) follow from $[20$, Theorem B]. We refer the reader to $[2,6,7,17]$ and references therein for more information on the study of the existence of stationary measures of a Fokker-Planck equation in general.

2.2. Stochastic stability. The following equivalence result on $\mathcal{A}$-stability is proved in $[20$, Proposition 3.4].

Lemma 2.2. Let $\Omega \subset \mathbb{R}^{n}$ be a compact invariant set of $\varphi^{t}$ and $\mathcal{A}=\left\{A_{\alpha}\right\} \subset \tilde{\mathcal{A}}$ be an admissible null family. Then $\Omega$ is $\mathcal{A}$-stable if and only if as $A_{\alpha} \rightarrow 0$, the set $\left\{\mu_{A_{\alpha}}\right\}$ of stationary measures corresponding to $\left\{\mathcal{L}_{A_{\alpha}}\right\}$ is relatively sequentially compact in $M\left(\mathbb{R}^{n}\right)$ and all $\mathcal{A}$-limit measures are supported on $\Omega$. 
For an admissible null family $\mathcal{A}$, denote

$$
\mathcal{J}_{\mathcal{A}}=\overline{\cup\{\operatorname{supp}(\mu): \mu \text { is an } \mathcal{A} \text {-limit measure }\}} .
$$

The following result follows from [20, Proposition $3.3 \mathrm{~b}$ )].

Lemma 2.3. (Stochastic LaSalle invariance principle) Let $\mathcal{A}$ be an admissible null family and suppose that $\mathcal{J}_{\mathcal{A}}$ is contained in a compact set $\mathcal{J}$ and (1.3) admits an entire weak Lyapunov function $U_{0}$ in a neighborhood of $\mathcal{J}$. Then any $\mathcal{A}$-limit measure is supported on the set $\left\{x \in \mathcal{J}: \nabla f(x) \cdot \nabla U_{0}(x)=0\right\}$.

We first give the following result concerning the stability of compact invariant sets of (1.3) under general noise perturbations.

Theorem 2.1. (Set stability under general noise) Consider (1.3) and assume that it admits a $C^{2}$ Lyapunov function whose second derivative is bounded. Then the following holds.

a) The set $E$ of critical points of $f$ is $\mathcal{A}$-stable with respect to any null family $\mathcal{A}$;

b) For any finite set $\mathcal{J}_{0}$ (resp. $\mathcal{R}_{0}$ ) of isolated local minimal (maximal) points of $f$, there is a null family $\mathcal{A}$ such that $\mathcal{J}_{0}$ (resp. $\mathcal{R}_{0}$ ) is $\mathcal{A}$-stable (resp. strongly $\mathcal{A}$-unstable).

c) Any simple local maximal point of $f$ is strongly $\mathcal{A}$-unstable with respect to any normal null family $\mathcal{A}$.

Proof. Let $\mathcal{A}=\left\{A_{\alpha}\right\}$ be a given null family. Since (1.3) admits a $C^{2}$ Lyapunov function $U$ whose second derivative is bounded, $U$ becomes a uniform Lyapunov function with respect to $\left\{\mathcal{L}_{A_{\alpha}}\right\}$ as $\left|A_{\alpha}\right| \ll 1$. By Lemma 2.1 a), $\mathcal{A}$ is admissible.

Let $\left\{\mu_{A_{\alpha}}\right\}$ denote the set of all stationary measures corresponding to $\left\{\mathcal{L}_{A_{\alpha}}\right\}$. It follows from Lemma $2.1 \mathrm{~b}$ ) that $\left\{\mu_{A_{\alpha}}\right\}$ is relatively sequentially compact in $M\left(\mathbb{R}^{n}\right)$ as $A_{\alpha} \rightarrow 0$, and from Lemma $2.1 \mathrm{c}$ ) that the set $\mathcal{J}_{\mathcal{A}}$ is contained in the global attractor $\mathcal{J}$ of $\varphi^{t}$. It is easy to see that there is a neighborhood of $\mathcal{J}$ and a constant $c>0$ such that $U_{0}=: f+c$ is an entire weak Lyapunov function of (1.3) in that neighborhood. It follows from Lemma 2.3 that any $\mathcal{A}$-limit measure is actually supported on the set

$$
\left\{x \in \mathcal{J}: \nabla f(x) \cdot \nabla U_{0}(x)=0\right\}=\left\{x \in \mathcal{J}:|\nabla f(x)|^{2}=0\right\}
$$

which is precisely the set $E$. By Lemma $2.2, E$ is $\mathcal{A}$-stable. This proves a).

b) follows from $\left[20\right.$, Theorem $\mathrm{C}$ a), b)] because the set $\mathcal{J}_{0}$ (resp. $\left.\mathcal{R}_{0}\right)$ is a strong local attractor (resp. repeller) of $\varphi^{t}$.

c) follows from [20, Theorem $\mathrm{C}$ c)] because each non-degenerate local maximal point of $f$ is a strongly repelling equilibrium of $\varphi^{t}$ in the sense of [20, Definition 6.4] when taking $U=f$ in that definition.

In the above theorem, a normal null family is a restricted null family defined in [20]. We now consider additive noise perturbation $\sqrt{2 \epsilon} \dot{W}$ to (1.3).

Lemma 2.4. Assume the condition $\mathbf{H})$. For each $\epsilon>0$, denote the Gibbs measure by $\mu_{\epsilon}$. Then the following holds. 
a) (Tightness) There exists an $\epsilon_{0}>0$, depending on $n$ and $\beta$, such that $\left\{\mu_{\epsilon}\right\}_{0<\epsilon \leq \epsilon_{0}}$ is relatively sequentially compact in $M\left(\mathbb{R}^{n}\right)$.

b) (Concentration) As $\epsilon \rightarrow 0$, any limit measure of $\left\{\mu_{\epsilon}\right\}$ is supported on the set $S_{f}$ of global minimal points of $f$.

In the above, a) follows from [21, Proposition 2.3] and b) follows from [21, Corollary 2.1]. We note that, under the condition $\mathbf{H}), f$ itself is a weak Lyapunov function of (1.3). Hence $\varphi^{t}$ becomes a semiflow on $\mathbb{R}^{n}$.

Theorem 2.2. (Stabilities under additive noise) Assume the condition $\mathbf{H})$. Then the following holds.

a) $S_{f}$ is $\mathcal{A}_{0}$-stable.

b) In the case $\mathbf{C 1})$, the measure $\delta_{*}=: \sum_{i=1}^{m} c_{i} \delta_{x_{i}}$ is $\mathcal{A}_{0}$-stable, where, for each $i=$ $1, \cdots, m$,

$$
c_{i}= \begin{cases}0, & \text { if } k_{i}<k, \\ \frac{\int_{\mathbb{R}^{n}} \mathrm{e}^{-P_{i}(y)} \mathrm{d} y}{\sum_{j: k_{j}=k} \int_{\mathbb{R}^{n}} \mathrm{e}^{-P_{j}(y)} \mathrm{d} y}, & \text { if } k_{i}=k,\end{cases}
$$

with $k=\max \left\{k_{i}: i=1,2, \cdots, m\right\}$.

c) In the case $\mathbf{C 2}$ ), the normalized Lebesgue measure $\mu_{f}$ on $S_{f}$ is $\mathcal{A}_{0}$-stable.

Proof. For each $\epsilon>0$, an application of [8, Example 5.4], with $A=\epsilon I$ and $b \equiv \beta_{\mu, A}$ there, shows the uniqueness of stationary measures corresponding to $\mathcal{L}_{\epsilon I}$. Hence the Gibbs measure $\mu_{\epsilon}$ is actually the unique stationary measure corresponding to $\mathcal{L}_{\epsilon I}$. Let $\mu$ be a limit point of $\left\{\mu_{\epsilon}\right\}$ as $\epsilon \rightarrow 0$. By Lemma $\left.2.4 \mathrm{~b}\right), \operatorname{supp}(\mu) \subset S_{f}$. It follows from Lemma 2.2 and Lemma 2.4 a) that $S_{f}$ is $\mathcal{A}_{0}$-stable. This proves a).

In the case C1), it follows from [3, Theorem 5.2] that $\lim _{\epsilon \rightarrow 0} \mu_{\epsilon}=\delta_{*}$, and in the case C2), it follows from [21, Proposition 2.2] that $\lim _{\epsilon \rightarrow 0} \mu_{\epsilon}=\mu_{f}$. This proves b) and c).

Remark 2.2. 1) The proof of [8, Example 5.4] actually implies that the Gibbs measure $\mu_{\epsilon}$, for a fixed $\epsilon>0$, is an invariant measure of the semigroup generated by the closure of the operator $\mathcal{L}_{\epsilon I}$ on $L^{1}\left(\mathbb{R}^{n}, \mu_{\epsilon}\right)$ (see also [5] for some interesting discussions in this regard). In fact, it can be further shown that if (1.4) generates a diffusion process then $\mu_{\epsilon}$ is an invariant measure of that process.

2) We would like to make some comments about the measure $\delta_{*}$ in Theorem 2.2. By the expression of $c_{i}$ in the above, we see that among the global minimal points of $f,\left\{\mu_{\epsilon}\right\}$ are eventually concentrated only on the most degenerate ones, i.e., those with the greatest order $2 k$ in the dominated terms of their Taylor expansions. If there are more than one most degenerate global minimal points, then $\left\{\mu_{\epsilon}\right\}$ are eventually concentrated on all these points with certain weights $c_{i}$ determined by the coefficients of $P_{i}$. For instance, consider the case $n=1$. Then $P_{i}(x)=A_{i} x^{2 k_{i}}$ for some $A_{i}>0$. Hence the Taylor expansion of $f$ in $B_{\delta}\left(x_{i}\right)$ reads

$$
f(x)=A_{i}\left(x-x_{i}\right)^{2 k_{i}}+o\left(\left|x-x_{i}\right|^{2 k_{i}}\right),
$$


from which it is not hard to see that

$$
c_{i}= \begin{cases}0, & \text { if } k_{i}<k \\ \frac{\sqrt[2 k]{\frac{1}{A_{i}}}}{\sum_{j: k_{j}=k} \sqrt[2 k]{\frac{1}{A_{j}}}}, & \text { if } k_{i}=k .\end{cases}
$$

Hence it is clear that $\left\{\mu_{\epsilon}\right\}$ are eventually concentrated with larger probability on those global minimal points having smaller coefficients $A_{i}$.

\section{An eXAmple of NON-CONVERGEnCE}

In this section, we construct an example of a potential function $f$ so that the set of stationary measures $\left\{\mu_{\epsilon}\right\}$ corresponding to $\left\{\mathcal{L}_{\epsilon I}\right\}$ admits more than one limit measure as $\epsilon \rightarrow 0$. In virtue of Theorem $2.2 \mathrm{~b}$ ), c), such a potential function thus satisfies neither condition in C1) nor condition in C2). Main features of this example have already been summarized in Theorem 1 of Section 1.

The construction of such a potential function is rather delicate. We first construct a "staircase" function with countable many steps accumulating at two absolute minimal points. The "staircase" function is then smoothed out to obtain the desired potential function whose corresponding Gibbs measures can be estimated by using the "staircase" function as an approximation. In this construction, the non-convergence property of the Gibbs measures is guaranteed by a careful selection of the jumping points of the "staircase" function.

The construction will be divided into several steps as follows.

Step 1. A simple computation shows that

$$
\begin{gathered}
\lim _{x \rightarrow+\infty} \sqrt{2}\left(\sum_{j=-\infty}^{+\infty} \mathrm{e}^{-\frac{1}{x^{2 j}}} \cdot \frac{1}{2^{j}}\right)=\sqrt{2}\left(\sum_{j=1}^{+\infty} \frac{1}{2^{j}}+\frac{1}{\mathrm{e}}\right) \\
=\sqrt{2}\left(1+\frac{1}{\mathrm{e}}\right)<2=\sum_{j=0}^{+\infty} \frac{1}{2^{j}}=\lim _{x \rightarrow+\infty} \sum_{j=-\infty}^{+\infty} \mathrm{e}^{-\frac{1}{x^{2 j+1}}} \cdot \frac{1}{2^{j}} .
\end{gathered}
$$

Thus there exists a constant $M_{*}>0$ such that for any $a \geq M_{*}$, if $A:=\sum_{j=-\infty}^{+\infty} \mathrm{e}^{-\frac{1}{a^{2 j}}} \cdot \frac{1}{2^{j}}$ and $B:=\sum_{j=-\infty}^{+\infty} \mathrm{e}^{-\frac{1}{a^{2 j+1}}} \cdot \frac{1}{2^{j}}$, then $\sqrt{2} A<B$, i.e., $\frac{A}{A+B}<\frac{B}{2 A+B}$.

Fix $a \geq \max \left\{8, M_{*}\right\}$. We first consider a measurable function $f(x), x \in \mathbb{R}$, defined in the following way:

For $x \in E_{1}=:\left(-\frac{3}{2},-\frac{1}{2}\right), f$ is a step function attaining minimal value at $x=-1$ and the graph of $f$ is symmetric with respect to the line $x=-1$. More precisely, for all $i \in \mathbb{N}$,

$$
\begin{aligned}
& f(-1+t)= \\
& \begin{cases}\frac{1}{a^{2 i}}, & t \in\left(\frac{1}{2}-\sum_{j=1}^{i} \frac{1}{2^{j+1}}, \frac{1}{2}-\sum_{j=1}^{i-1} \frac{1}{2^{j+1}}\right] \cup\left[-\frac{1}{2}+\sum_{j=1}^{i-1} \frac{1}{2^{j+1}},-\frac{1}{2}+\sum_{j=1}^{i} \frac{1}{2^{j+1}}\right), \\
0, & t=0 .\end{cases}
\end{aligned}
$$

For $x \in E_{2}=:\left(\frac{1}{2}, \frac{3}{2}\right), f$ has a similar shape, i.e., for all $i \in \mathbb{N}$,

$$
f(1+t)=
$$




$$
\begin{cases}\frac{1}{a^{2 i+1}}, & t \in\left(\frac{1}{2}-\sum_{j=1}^{i} \frac{1}{2^{j+1}}, \frac{1}{2}-\sum_{j=1}^{i-1} \frac{1}{2^{j+1}}\right] \cup\left[-\frac{1}{2}+\sum_{j=1}^{i-1} \frac{1}{2^{j+1}},-\frac{1}{2}+\sum_{j=1}^{i} \frac{1}{2^{j+1}}\right), \\ 0, & t=0 .\end{cases}
$$

On the set $E=: \mathbb{R} \backslash\left(E_{1} \cup E_{2}\right), f$ is $C^{\infty}$ smooth and smoothly connecting at the points $-\frac{3}{2},-\frac{1}{2}, \frac{1}{2}, \frac{3}{2}$. Moreover, $f(x) \geq \frac{1}{a^{3}}$ on $E$, and, $f(x)=x^{2}$ when $|x| \gg 1$.

We refer the reader to Figure 1 for a sketch of the graph of $f$.

For each $i \in \mathbb{N}$, we let $\varepsilon_{i}=\frac{1}{a^{2 i}}, \delta_{i}=\frac{1}{a^{2 i-1}}$, and denote by $\mu_{i}, \nu_{i}$ the Gibbs measures associated with $u_{\varepsilon_{i}}=: \frac{\mathrm{e}^{-\frac{f}{\varepsilon_{i}}}}{\int_{\mathbb{R}} \mathrm{e}^{-\frac{f}{\varepsilon_{i}}} \mathrm{~d} x}, u_{\delta_{i}}=: \frac{\mathrm{e}^{-\frac{f}{\delta_{i}}}}{\int_{\mathbb{R}} \mathrm{e}^{-\frac{f}{\delta_{i}}} \mathrm{~d} x}$, respectively; i.e., $\mathrm{d} \mu_{i}(x)=u_{\varepsilon_{i}}(x) \mathrm{d} x$, $\mathrm{d} \nu_{i}(x)=u_{\delta_{i}}(x) \mathrm{d} x$. Then, as $i \rightarrow+\infty$, we have

$$
\begin{aligned}
\mu_{i}\left(E_{1}\right) & =\frac{\sum_{j=1}^{\infty} \mathrm{e}^{-\frac{1}{a^{2 j} \varepsilon_{i}}} \cdot \frac{1}{2^{j}}}{\sum_{j=1}^{\infty} \mathrm{e}^{-\frac{1}{a^{2 j} \varepsilon_{i}}} \cdot \frac{1}{2^{j}}+\sum_{j=1}^{\infty} \mathrm{e}^{-\frac{1}{a^{2 j+1} \varepsilon_{i}}} \cdot \frac{1}{2^{j}}+\int_{E} \mathrm{e}^{-\frac{f(x)}{\varepsilon_{i}}} \mathrm{~d} x} \\
& =\frac{\sum_{j=1}^{\infty} \mathrm{e}^{-\frac{1}{a^{2(j-i)}} \cdot \frac{1}{2^{j}}}}{\sum_{j=1}^{\infty} \mathrm{e}^{-\frac{1}{a^{2(j-i)}}} \cdot \frac{1}{2^{j}}+\sum_{j=1}^{\infty} \mathrm{e}^{-\frac{1}{a^{2(j-i)+1}}} \cdot \frac{1}{2^{j}}+\int_{E} \mathrm{e}^{-\frac{f(x)}{\varepsilon_{i}}} \mathrm{~d} x} \\
& =\frac{\frac{1}{2^{i}} \sum_{j=1-i}^{\infty} \mathrm{e}^{-\frac{1}{a^{2 j}}} \cdot \frac{1}{2^{j}}}{\frac{1}{2^{i}} \sum_{j=1-i}^{\infty} \mathrm{e}^{-\frac{1}{a^{2 j}}} \cdot \frac{1}{2^{j}}+\frac{1}{2^{i}} \sum_{j=1-i}^{\infty} \mathrm{e}^{-\frac{1}{a^{2 j}+1}} \cdot \frac{1}{2^{j}}+\int_{E} \mathrm{e}^{-\frac{f(x)}{\varepsilon_{i}}} \mathrm{~d} x} \\
& =\frac{\sum_{j=1-i}^{\infty} \mathrm{e}^{-\frac{1}{a^{2 j}}} \cdot \frac{1}{2^{j}}}{\sum_{j=1-i}^{\infty} \mathrm{e}^{-\frac{1}{a^{2 j}}} \cdot \frac{1}{2^{j}}+\sum_{j=1-i}^{\infty} \mathrm{e}^{-\frac{1}{a^{2 j}+1}} \cdot \frac{1}{2^{j}}+2^{i} \int_{E} \mathrm{e}^{-\frac{f(x)}{\varepsilon_{i}}} \mathrm{~d} x} \\
& \longrightarrow \frac{A}{A+B},
\end{aligned}
$$

and

$$
\begin{aligned}
\nu_{i}\left(E_{1}\right) & =\frac{\sum_{j=1}^{\infty} \mathrm{e}^{-\frac{1}{a^{2 j} \delta_{i}} \cdot \frac{1}{2^{j}}}}{\sum_{j=1}^{\infty} \mathrm{e}^{-\frac{1}{a^{2 j} \delta_{i}}} \cdot \frac{1}{2^{j}}+\sum_{j=1}^{\infty} \mathrm{e}^{-\frac{1}{a^{2 j}+1} \delta_{i}} \cdot \frac{1}{2^{j}}+\int_{E} \mathrm{e}^{-\frac{f(x)}{\delta_{i}}} \mathrm{~d} x} \\
& =\frac{\sum_{j=1}^{\infty} \mathrm{e}^{-\frac{1}{a^{2(j-i)+1}} \cdot \frac{1}{2^{j}}}}{\sum_{j=1}^{\infty} \mathrm{e}^{-\frac{1}{a^{2(j-i)+1}}} \cdot \frac{1}{2^{j}}+\sum_{j=1}^{\infty} \mathrm{e}^{-\frac{1}{a^{2(j-i+1)}}} \cdot \frac{1}{2^{j}}+\int_{E} \mathrm{e}^{-\frac{f(x)}{\delta_{i}}} \mathrm{~d} x} \\
& =\frac{\frac{1}{2^{i}} \sum_{j=1-i}^{\infty} \mathrm{e}^{-\frac{1}{a^{2 j}+1}} \cdot \frac{1}{2^{j}}}{\frac{1}{2^{i}} \sum_{j=1-i}^{\infty} \mathrm{e}^{-\frac{1}{a^{2 j}+1}} \cdot \frac{1}{2^{j}}+\frac{1}{2^{i-1}} \sum_{j=2-i}^{\infty} \mathrm{e}^{-\frac{1}{a^{2 j}}} \cdot \frac{1}{2^{j}}+\int_{E} \mathrm{e}^{-\frac{f(x)}{\delta_{i}}} \mathrm{~d} x} \\
& =\frac{\sum_{j=1-i}^{\infty} \mathrm{e}^{-\frac{1}{a^{2 j}+1}} \cdot \frac{1}{2^{j}}}{\sum_{j=1-i}^{\infty} \mathrm{e}^{-\frac{1}{a^{2 j+1}}} \cdot \frac{1}{2^{j}}+2 \sum_{j=2-i}^{\infty} \mathrm{e}^{-\frac{1}{a^{2 j}}} \cdot \frac{1}{2^{j}}+2^{i} \int_{E} \mathrm{e}^{-\frac{f(x)}{\delta_{i}}} \mathrm{~d} x} \\
& \longrightarrow \frac{B}{2 A+B} .
\end{aligned}
$$

Step 2. Fix two $C^{\infty}$ functions $g_{1}, g_{2}: \mathbb{R} \rightarrow[0,1]$ satisfying

$$
g_{1}(x)=\left\{\begin{array}{ll}
0, & \text { for } x \leq 0, \\
1, & \text { for } x \geq 1,
\end{array} \quad g_{2}(x)= \begin{cases}1, & x \leq 0 \\
0, & x \geq 1\end{cases}\right.
$$


and $\sup _{x \in \mathbb{R}} \max \left\{\left|g_{1}^{\prime}(x)\right|,\left|g_{2}^{\prime}(x)\right|,\left|g_{1}{ }^{\prime \prime}(x)\right|,\left|g_{2}{ }^{\prime \prime}(x)\right|\right\} \leq M$ for some constant $M>0$. For given $L, R, z \in \mathbb{R}, d>0$ with $L \neq R$, consider the function $g_{L, R, d, z}: \mathbb{R} \rightarrow[\min \{L, R\}, \max \{L, R\}]$ :

$$
g_{L, R, d, z}(x)= \begin{cases}(R-L) g_{1}\left(\frac{x-z}{d}\right)+L, & \text { when } L<R ; \\ (L-R) g_{2}\left(\frac{x-z}{d}\right)+R, & \text { when } L>R .\end{cases}
$$

Then it is clear that

$$
g_{L, R, d, z}(x)= \begin{cases}L, & x \leq z, \\ R, & x \geq z+d,\end{cases}
$$

$\left|g_{L, R, d, z}^{\prime}(x)\right| \leq \frac{|R-L|}{d} M$, and $\left|g^{\prime \prime}{ }_{L, R, d, z}(x)\right| \leq \frac{|R-L|}{d^{2}} M$.

For a fixed small constant $\eta>0$, we now use the function $g_{L, R, d, z}$ to construct a function $f_{\eta} \in C^{2}(\mathbb{R})$ satisfying the following properties:

P1) $f_{\eta} \equiv f$ on $E$ and $f_{\eta} \geq f$ on $E_{i}, i=1,2$;

P2) $f_{\eta}$ attains global minima at $\{ \pm 1\}$ which are however not isolated critical points of $f_{\eta}$;

P3) For each constant $c \geq 0$,

$$
\int_{E_{i}} \mathrm{e}^{-c f_{\eta}} \mathrm{d} x \geq(1-\eta) \int_{E_{i}} \mathrm{e}^{-c f} \mathrm{~d} x, \quad i=1,2 .
$$

The construction is a smoothing process. At each point of discontinuity $z=-1-\frac{1}{2}+$ $\sum_{j=1}^{i} \frac{1}{2^{j+1}} \in\left(-\frac{3}{2},-1\right)$, we let $L=\frac{1}{a^{2 i}}, R=\frac{1}{a^{2(i+1)}}$, and $d=\eta \cdot \frac{1}{2^{i+2}}$; at each point of discontinuity $z=-1+\frac{1}{2}-\sum_{j=1}^{i} \frac{1}{2^{j+1}} \in\left(-1,-\frac{1}{2}\right)$, we let $L=\frac{1}{a^{2(i+1)}}, R=\frac{1}{a^{2 i}}$, and $d=\eta \cdot \frac{1}{2^{i+2}}$; at each point of discontinuity $z=1-\frac{1}{2}+\sum_{j=1}^{i} \frac{1}{2^{j+1}} \in\left(\frac{1}{2}, 1\right)$, we let $L=\frac{1}{a^{2 i+1}}, R=\frac{1}{a^{2(i+1)+1}}$, and $d=\eta \cdot \frac{1}{2^{i+2}}$; and at each point of discontinuity $z=1+\frac{1}{2}-\sum_{j=1}^{i} \frac{1}{2^{j+1}} \in\left(1, \frac{3}{2}\right)$, we let $L=\frac{1}{a^{2(i+1)+1}}, R=\frac{1}{a^{2 i+1}}$, and $d=\eta \cdot \frac{1}{2^{i+2}}$. We let $f_{\eta}(x)=g_{L, R, d, z}(x)$ for $x$ lying in each interval of the form $[z, z+d] \subset\left(-\frac{3}{2},-1\right) \cup\left(\frac{1}{2}, 1\right), f_{\eta}(x)=g_{L, R, d, z-d}(x)$ for $x$ lying in each interval of the form $[z-d, z] \subset\left(-1,-\frac{1}{2}\right) \cup\left(1, \frac{3}{2}\right)$, and $f_{\eta}(x)=f(x)$ for $x$ lying in the complement of these intervals.

The function $f_{\eta}$ is $C^{\infty}$ everywhere just with possible exception at the points $x=-1$ and $x=1$. Note that when $x \in\left[-\frac{3}{2}+\sum_{j=1}^{i} \frac{1}{2^{j+1}},-\frac{3}{2}+\sum_{j=1}^{i} \frac{1}{2^{j+1}}+\eta \cdot \frac{1}{2^{i+2}}\right]$, we have $\frac{1}{2^{i+2}} \leq|x-(-1)| \leq \frac{1}{2^{i+1}}$. Using the fact $a \geq 8$, it follows that, when $|x-(-1)| \ll 1$,

$$
\begin{aligned}
& \left|f_{\eta}^{\prime}(x)\right| \leq \frac{|L-R|}{d} M \leq \frac{\frac{1}{a^{2 i}}}{\eta \cdot \frac{1}{2^{i+2}}} M=\frac{M 2^{4(i+2)}}{\eta a^{2 i}} \cdot \frac{1}{2^{3(i+2)}} \leq \frac{M}{\eta}|x-(-1)|^{3}, \\
& \left|f_{\eta}^{\prime \prime}(x)\right| \leq \frac{|L-R|}{d^{2}} M \leq \frac{\frac{1}{a^{2 i}}}{\eta^{2} \cdot \frac{1}{2^{2(i+2)}}} M=\frac{16 M}{\eta^{2}}\left(\frac{2}{a}\right)^{2 i} \frac{2^{2(i+2)}}{2^{2(i+2)}} \leq \frac{M}{\eta^{2}}|x-(-1)|^{2} .
\end{aligned}
$$

Therefore $f_{\eta}^{\prime}(-1-0)=0$ and ${f^{\prime \prime}}_{\eta}(-1-0)=0$. Similarly, $f_{\eta}^{\prime}(-1+0)=0$ and $f^{\prime \prime} \eta(-1+0)=0$. Thus both $f_{\eta}^{\prime}(-1)$ and $f_{\eta}^{\prime \prime}(-1)$ exist and equal 0 . By the definition of $f_{\eta}$, we have

$$
\left|f_{\eta}(x)-f_{\eta}(-1)\right|=\left|f_{\eta}(x)\right| \leq \frac{1}{a^{2 i}} \leq|x-(-1)|^{4}
$$

when $|x-(-1)| \ll 1$. Hence $f_{\eta}^{\prime}$ is continuous at -1 . Since

$$
\left|f_{\eta}^{\prime}(x)-f_{\eta}^{\prime}(-1)\right|=\left|f_{\eta}^{\prime}(x)\right| \leq \frac{M}{\eta}|x-(-1)|^{3}
$$

when $|x-(-1)| \ll 1, f_{\eta}{ }^{\prime \prime}$ is continuous at -1 . The same arguments show that $f_{\eta}^{\prime}(1), f_{\eta}{ }^{\prime \prime}(1)$ exist, both equal 0 , and $f_{\eta}^{\prime}, f_{\eta}^{\prime \prime}$ are continuous at 1 . 
It is clear that $f_{\eta}$ so constructed satisfies all properties P1)-P3) above.

Step 3. Consider the noise perturbed gradient system

$$
\dot{x}=-f_{\eta}^{\prime}(x)+\sqrt{2 \epsilon} \dot{W}, \quad x \in \mathbb{R}^{1} .
$$

We note that with $f_{\eta}$ being a weak Lyapunov function the unperturbed gradient system corresponding to (3.1) generates a semiflow on $\mathbb{R}^{1}$, which we denote by $\varphi^{t}$.

For each $\epsilon>0$, an application of [8, Example 5.4] shows that the Gibbs state

$$
u_{\epsilon}^{\eta}(x)=\frac{\mathrm{e}^{-\frac{f_{\eta}(x)}{\epsilon}}}{\int_{\mathbb{R}} \mathrm{e}^{-\frac{f_{\eta}(x)}{\epsilon}} \mathrm{d} x}
$$

is the unique stationary solution of the Fokker-Planck equation associated with (3.1). This also follows from [8, Example 5.1] because $U(x)=: f_{\eta}(x)=x^{2}$ as $|x| \gg 1$, is an unbounded, uniform Lyapunov function with respect to the family $\left\{\mathcal{L}_{\epsilon I}\right\}$ of the adjoint Fokker-Planck operators corresponding to (3.1) (see Remark 2.1). By either Lemma 2.1 b) or Lemma 2.4 a), the set of limit measures of the corresponding Gibbs measures as $\epsilon \rightarrow 0$ is non-empty.

For each $i \in \mathbb{N}$, we let $\varepsilon_{i}=\frac{1}{a^{2 i}}, \delta_{i}=\frac{1}{a^{2 i-1}}$ be the sequences used in Step 1 and let $\mu_{i}^{\eta}, \nu_{i}^{\eta}$ be the stationary measures associated with the Gibbs states $u_{\varepsilon_{i}}^{\eta}, u_{\delta_{i}}^{\eta}$, respectively. Choose any limit measure $\mu$ of $\left\{\mu_{i}^{\eta}\right\}$ and any limit measure $\nu$ of $\left\{\nu_{i}^{\eta}\right\}$, as $i \rightarrow \infty$. We have by Theorem 2.2 a) that both $\mu$ and $\nu$ are invariant measures of $\varphi^{t}$ supported on $S_{f_{\eta}}=\{-1,1\}$.

Using the properties P1)-P3) of $f_{\eta}$, it is easy to see that, as $i \rightarrow \infty$,

$$
\begin{aligned}
\mu_{i}^{\eta}\left(E_{1}\right) & =\frac{\int_{E_{1}} \mathrm{e}^{-f_{\eta} \cdot a^{2 i}} \mathrm{~d} x}{\int_{E_{1}} \mathrm{e}^{-f_{\eta} \cdot a^{2 i}} \mathrm{~d} x+\int_{E_{2}} \mathrm{e}^{-f_{\eta} \cdot a^{2 i}} \mathrm{~d} x+\int_{E} \mathrm{e}^{-f_{\eta} \cdot a^{2 i}} \mathrm{~d} x} \\
\leq & \frac{\int_{E_{1}} \mathrm{e}^{-f \cdot a^{2 i}} \mathrm{~d} x}{(1-\eta) \int_{E_{1}} \mathrm{e}^{-f \cdot a^{2 i}} \mathrm{~d} x+(1-\eta) \int_{E_{2}} \mathrm{e}^{-f \cdot a^{2 i}} \mathrm{~d} x+\int_{E} \mathrm{e}^{-f \cdot a^{2 i}} \mathrm{~d} x} \\
& \longrightarrow \frac{1}{(1-\eta)} \cdot \frac{A}{A+B}, \\
\nu_{i}^{\eta}\left(E_{1}\right) & =\frac{\int_{E_{1}} \mathrm{e}^{-f_{\eta} \cdot a^{2 i-1}} \mathrm{~d} x}{\int_{E_{1}} \mathrm{e}^{-f_{\eta} \cdot a^{2 i-1}} \mathrm{~d} x+\int_{E_{2}} \mathrm{e}^{-f_{\eta} \cdot a^{2 i-1}} \mathrm{~d} x+\int_{E} \mathrm{e}^{-f_{\eta} \cdot a^{2 i-1}} \mathrm{~d} x} \\
& \geq \frac{(1-\eta) \int_{E_{1}} \mathrm{e}^{-f \cdot a^{2 i-1}} \mathrm{~d} x}{\int_{E_{1}} \mathrm{e}^{-f \cdot a^{2 i-1}} \mathrm{~d} x+\int_{E_{2}} \mathrm{e}^{-f \cdot a^{2 i-1}} \mathrm{~d} x+\int_{E} \mathrm{e}^{-f \cdot a^{2 i-1}} \mathrm{~d} x} \\
& \longrightarrow(1-\eta) \cdot \frac{B}{2 A+B} .
\end{aligned}
$$

It follows that

$$
\mu\left(E_{1}\right) \leq \frac{1}{(1-\eta)} \cdot \frac{A}{A+B}<(1-\eta) \cdot \frac{B}{2 A+B} \leq \nu\left(E_{1}\right)
$$

when $\eta$ is sufficiently small. Hence $\mu \neq \nu$.

This shows that the family $\left\{\mu_{\epsilon}^{\eta}\right\}$ of all stationary measures corresponding to $\left\{\mathcal{L}_{\epsilon I}\right\}$ admits at least two limit measures as $\epsilon \rightarrow 0$. Consequently, no invariant measure of $\varphi^{t}$ can be $\{\epsilon I\}$-stable. 
We now show that $\mu, \nu$ have the desired forms. Since both $\mu$ and $\nu$ are supported on $S_{f_{\eta}}=\{-1,1\}$ and $\mu \neq \nu, \mu=\lambda_{1} \delta_{-1}+\left(1-\lambda_{1}\right) \delta_{1}$ and $\nu=\lambda_{2} \delta_{-1}+\left(1-\lambda_{2}\right) \delta_{1}$ for some constants $\lambda_{1} \neq \lambda_{2}$. We note that, as $i \rightarrow \infty$,

$$
\begin{aligned}
\mu_{i}^{\eta}\left(E_{1}\right) & =\frac{\int_{E_{1}} \mathrm{e}^{-f_{\eta} \cdot a^{2 i}} \mathrm{~d} x}{\int_{E_{1}} \mathrm{e}^{-f_{\eta} \cdot a^{2 i}} \mathrm{~d} x+\int_{E_{2}} \mathrm{e}^{-f_{\eta} \cdot a^{2 i}} \mathrm{~d} x+\int_{E} \mathrm{e}^{-f_{\eta} \cdot a^{2 i}} \mathrm{~d} x} \\
& \geq \frac{(1-\eta) \cdot \int_{E_{1}} \mathrm{e}^{-f \cdot a^{2 i}} \mathrm{~d} x}{\int_{E_{1}} \mathrm{e}^{-f \cdot a^{2 i}} \mathrm{~d} x+\int_{E_{2}} \mathrm{e}^{-f \cdot a^{2 i}} \mathrm{~d} x+\int_{E} \mathrm{e}^{-f \cdot a^{2 i}} \mathrm{~d} x} \\
& \longrightarrow(1-\eta) \cdot \frac{A}{A+B},
\end{aligned}
$$

and

$$
\begin{aligned}
\nu_{i}^{\eta}\left(E_{1}\right) & =\frac{\int_{E_{1}} \mathrm{e}^{-f_{\eta} \cdot a^{2 i-1}} \mathrm{~d} x}{\int_{E_{1}} \mathrm{e}^{-f_{\eta} \cdot a^{2 i-1}} \mathrm{~d} x+\int_{E_{2}} \mathrm{e}^{-f_{\eta} \cdot a^{2 i-1}} \mathrm{~d} x+\int_{E} \mathrm{e}^{-f_{\eta} \cdot a^{2 i-1}} \mathrm{~d} x} \\
& \leq \frac{\int_{E_{1}} \mathrm{e}^{-f \cdot a^{2 i-1}} \mathrm{~d} x}{(1-\eta) \cdot \int_{E_{1}} \mathrm{e}^{-f \cdot a^{2 i-1}} \mathrm{~d} x+(1-\eta) \cdot \int_{E_{2}} \mathrm{e}^{-f \cdot a^{2 i-1}} \mathrm{~d} x+\int_{E} \mathrm{e}^{-f_{\eta} \cdot a^{2 i-1}} \mathrm{~d} x} \\
& \longrightarrow \frac{1}{1-\eta} \cdot \frac{B}{2 A+B} .
\end{aligned}
$$

It follows that

$$
\mu\left(E_{1}\right) \geq(1-\eta) \cdot \frac{A}{A+B} \quad \text { and } \quad \nu\left(E_{1}\right) \leq \frac{1}{1-\eta} \cdot \frac{B}{2 A+B},
$$

which, together with $(3.2)$, yields that $\lambda_{1}, \lambda_{2} \in(0,1)$ when $\eta$ is small. This completes the proof.

\section{De-Stabilization of normalized Lebesgue measure via multiplicative noises}

For stochastic stability of singular measures, we have seen from Theorem 2.2 that those concentrated on relative minimal points of $f$ are never stable under additive noise perturbations, though they can be stable under some multiplicative noise perturbations according to Theorem $2.1 \mathrm{~b}$ ).

The situation with absolutely continuous measures is more complicated. We see from Theorem $2.2 \mathrm{c}$ ) that if the condition in C2) holds, then the normalized Lebesgue measure on $S_{f}$ is stable under additive noise perturbations. Below we give an example of a gradient system satisfying the condition in C2) for which there are infinitely many null families $\{\mathcal{A}\}$ of multiplicative noise perturbations such that with respect to each family $\mathcal{A}$ the set $S_{f}$ remains $\mathcal{A}$-stable but any $\mathcal{A}$-limit measure differs from the normalized Lebesgue measure on $S_{f}$, i.e., the normalized Lebesgue measure is $\mathcal{A}$-unstable. In fact, this example contains more information than that stated in Theorem 2.

Consider (1.3) with $n=1$ for a potential function $f \in C^{2}(\mathbb{R})$ such that

$$
f(x)= \begin{cases}0, & |x| \leq 1 \\ x^{2}, & |x| \geq 2,\end{cases}
$$

and $f(x)>0, f^{\prime}(x) \neq 0,1<|x|<2$. It is clear that $E=S_{f}$ which is precisely the closed interval $[-1,1]$. We note that $f$ itself is an unbounded $C^{2}$ Lyapunov function of (1.3) whose 
second derivatives are bounded on $\mathbb{R}$. Hence for any null family $\mathcal{A}=\left\{A_{\alpha}\right\} \subset \tilde{\mathcal{A}}, f$ is a uniform Lyapunov function with respect to $\left\{\mathcal{L}_{A_{\alpha}}\right\}$ and it follows from Theorem 2.1 a) that $S_{f}$ is $\mathcal{A}$-stable.

Now consider the noise perturbed equation

$$
\mathrm{d} x=-f^{\prime}(x) \mathrm{d} t+\sqrt{2 \epsilon a(x)} \mathrm{d} W, \quad x \in \mathbb{R},
$$

where $a \in C^{2}(\mathbb{R})$ is a bounded positive function. We then obtain the stationary Fokker-Planck equation

$$
\left\{\begin{array}{l}
\epsilon(a u)^{\prime \prime}+\left(u f^{\prime}\right)^{\prime}=0, \\
u(x) \geq 0, \quad \int_{-\infty}^{+\infty} u(x) \mathrm{d} x=1 .
\end{array}\right.
$$

Since $f$ is an unbounded uniform Lyapunov function with respect to the family $\left\{\mathcal{L}_{\epsilon a}\right\}$, Lemma 2.1, together with [8, Example 5.1], implies that, for each $a$ and $\epsilon>0$, (4.1) admits a unique classical, positive solution $u_{\epsilon}^{a}$ which gives rise to a stationary measure $\mu_{\epsilon}^{a}$ corresponding to $\mathcal{L}_{\epsilon a}$ with density $u_{\epsilon}^{a}$. Moreover, by Lemma $2.1 \mathrm{~b}$ ), the set of limit measures of $\left\{\mu_{\epsilon}^{a}\right\}$ as $\epsilon \rightarrow 0$ is non-empty.

By direct integrating, we obtain that $u_{\epsilon}^{a}$ has the following form:

$$
u_{\epsilon}^{a}(x)=\frac{N}{2 \epsilon a(x)} \mathrm{e}^{-\int_{0}^{x} \frac{f^{\prime}(x)}{\epsilon a(x)} \mathrm{d} x},
$$

with $N$ being the normalizing constant. Since $f^{\prime}(x)=0$ for $x \in[-1,1]$, we have

$$
\frac{\int_{a_{1}}^{b_{1}} a \mathrm{~d} \mu_{\epsilon}^{a}}{\int_{a_{2}}^{b_{2}} a \mathrm{~d} \mu_{\epsilon}^{a}}=\frac{\int_{a_{1}}^{b_{1}} a u_{\epsilon}^{a} \mathrm{~d} x}{\int_{a_{2}}^{b_{2}} a u_{\epsilon}^{a} \mathrm{~d} x}=\frac{b_{1}-a_{1}}{b_{2}-a_{2}}
$$

for any interval $\left[a_{i}, b_{i}\right] \subset[-1,1], i=1,2$. Let $\mu^{a}$ be any limit measure of $\left\{\mu_{\epsilon}^{a}\right\}$ as $\epsilon \rightarrow 0$. Then the same ratio formula holds with $\mu^{a}$ in place of $\mu_{\epsilon}^{a}$ in the above. Consequently, we obtain the following properties immediately:

(i) Each $\mu^{a}$ is supported on $S_{f}=[-1,1]$ but not on any proper compact subset of it.

(ii) By varying $a$, we obtain infinitely many limit measures different from the normalized Lebesgue measure on $S_{f}$.

Remark 4.1. The phenomena described by the above example are actually valid in any dimension. Consider the the noise perturbed system

$$
\mathrm{d} x=-\nabla f(x) \mathrm{d} t+\sqrt{2 \epsilon a(x)} I \mathrm{~d} W, \quad x \in \mathbb{R}^{n},
$$

where $f \in C^{2}\left(\mathbb{R}^{n}\right)$ is such that

$$
f(x)=\left\{\begin{array}{lr}
0, & |x| \leq 1 \\
|x|^{2}, & |x| \geq 2,
\end{array}\right.
$$

$f(x)>0$ and $\nabla f(x) \neq 0$ when $1<|x|<2$, and $a \in C^{2}\left(\mathbb{R}^{n}\right)$ is a bounded positive function.

The corresponding stationary Fokker-Planck equation reads

$$
\left\{\begin{array}{l}
\epsilon \Delta(a u)+\nabla \cdot(u \nabla f)=0, \\
u(x) \geq 0, \quad \int_{\mathbb{R}^{n}} u(x) \mathrm{d} x=1 .
\end{array}\right.
$$


Consider the function

$$
f_{\epsilon}(\rho):=\rho^{1-n} \int_{\partial B_{\rho}} a u_{\epsilon}^{a} \mathrm{~d} s,
$$

where $B_{\rho}$ is the ball with radius $\rho$ centered at the origin for each $\rho \in(0,1]$. Using the radial coordinate $r=|x|$ and letting $\omega=\frac{x}{r}$, we have $u_{\epsilon}^{a}(x)=u_{\epsilon}^{a}(r \omega)$. Moreover,

$$
\begin{aligned}
\int_{\partial B_{\rho}} \frac{\partial\left(a u_{\epsilon}^{a}\right)}{\partial \vec{n}} \mathrm{~d} s & =\int_{\partial B_{\rho}} \frac{\partial\left(a u_{\epsilon}^{a}\right)}{\partial r}(\rho \omega) \mathrm{d} s=\rho^{n-1} \int_{|\omega|=1} \frac{\partial\left(a u_{\epsilon}^{a}\right)}{\partial r}(\rho \omega) \mathrm{d} \omega \\
& =\rho^{n-1} \frac{\partial}{\partial \rho} \int_{|\omega|=1}\left(a u_{\epsilon}^{a}\right)(\rho \omega) \mathrm{d} \omega=\rho^{n-1} \frac{\partial}{\partial \rho}\left(\rho^{1-n} \int_{\partial B_{\rho}}\left(a u_{\epsilon}^{a}\right)(x) \mathrm{d} s\right),
\end{aligned}
$$

i.e.,

$$
f_{\epsilon}^{\prime}(\rho)=\rho^{1-n} \int_{\partial B_{\rho}} \frac{\partial\left(a u_{\epsilon}^{a}\right)}{\partial \vec{n}} \mathrm{~d} s .
$$

But the Divergence Theorem together with (4.2) imply that

$$
\int_{\partial B_{\rho}} \frac{\partial\left(a u_{\epsilon}^{a}\right)}{\partial \vec{n}} \mathrm{~d} s=\int_{\partial B_{\rho}} \nabla\left(a u_{\epsilon}^{a}\right) \cdot \vec{n} \mathrm{~d} s=\int_{B_{\rho}} \Delta\left(a u_{\epsilon}^{a}\right) \mathrm{d} x=-\frac{1}{\epsilon} \int_{B_{\rho}} \nabla \cdot\left(u_{\epsilon}^{a} \nabla f\right) \mathrm{d} x=0 .
$$

Hence

$$
f_{\epsilon}^{\prime}(\rho)=0, \quad \text { for all } \rho \in(0,1] .
$$

It follows that there is a positive constant $C$, depending on $a$ and $\epsilon$, such that

$$
\int_{\partial B_{\rho}} a u_{\epsilon}^{a} \mathrm{~d} s=C \rho^{n-1}, \quad \text { for all } \rho \in(0,1]
$$

from which we deduce that

$$
\int_{B_{r}} a u_{\epsilon}^{a} \mathrm{~d} x=\int_{0}^{r} \int_{\partial B_{\rho}} a u_{\epsilon}^{a} \mathrm{~d} s \mathrm{~d} \rho=\int_{0}^{r} C \rho^{n-1} \mathrm{~d} \rho=\frac{1}{n} C r^{n},
$$

for any $0<r \leq 1$. Thus, for any $0<r<R \leq 1$, we have

$$
\frac{\int_{B_{r}} a \mathrm{~d} \mu_{\epsilon}^{a}}{\int_{B_{R} \backslash B_{r}} a \mathrm{~d} \mu_{\epsilon}^{a}}=\frac{\int_{B_{r}} a u_{\epsilon}^{a} \mathrm{~d} x}{\int_{B_{R} \backslash B_{r}} a u_{\epsilon}^{a} \mathrm{~d} x}=\frac{r^{n}}{R^{n}-r^{n}} .
$$

Let $\mu^{a}$ be any limit measure of $\left\{\mu_{\epsilon}^{a}\right\}$ as $\epsilon \rightarrow 0$. Then

$$
\frac{\int_{B_{r}} a \mathrm{~d} \mu^{a}}{\int_{B_{R} \backslash B_{r}} a \mathrm{~d} \mu^{a}}=\frac{r^{n}}{R^{n}-r^{n}}
$$

for any $0<r<R \leq 1$. The following properties follow immediately from (4.3):

(i) Each $\mu^{a}$ is supported on $\bar{B}_{1}$ but not on any proper compact subset of it.

(ii) For any two fixed number $0<r<R \leq 1$ and any two functions $a_{1}, a_{2}$ with $a_{1}(x)<$ $a_{2}(x), x \in B_{r} ; a_{1}(x)=a_{2}(x), x \in \partial B_{r} ;$ and $a_{1}(x)>a_{2}(x), x \in B_{R} \backslash \bar{B}_{r}$, any limit measure $\mu^{a_{1}}$ corresponding to $a_{1}$ is different from any limit measure $\mu^{a_{2}}$ corresponding to $a_{2}$.

In particular, by varying $a$, we obtain infinitely many limit measures differing from the normalized Lebesgue measure on $\bar{B}_{1}=S_{f}$. 


\section{REFERENCES}

[1] P. Ao, H. Qian, Y. Tu, and J. Wang, A theory of mesoscopic phenomena: Time scales, emergent unpredictability, symmetry breaking and dynamics across different levels, preprint 2013.

[2] A. Arapostathis, V. S. Borkar, and M. K. Ghosh, Ergodic Control of Diffusion Processes, Cambridge University Press, Cambridge, 2012.

[3] K. B. Athreya, and C.-R. Hwang, Gibbs measures asymptotics, Sankhya A 72 (2010), 191-207.

[4] V. I. Bogachev, N. V. Krylov, and M. Röckner, On regularity of transition probabilities and invariant measures of singular diffusions under minimal conditions, Comm. Partial Differential Equations 26 (2001), 2037-2080.

[5] V. I. Bogachev, N. V. Krylov, and M. Röckner, Elliptic and parabolic equations for measures, Russian Math. Surveys 64 (6) (2009), 973-1078.

[6] V. I. Bogachev and M. Röckner, A generalization of Khasminskii's theorem on the existence of invariant measures for locally integrable drifts, Theory Probab. Appl. 45 (2001), 363-378.

[7] V. I. Bogachev, M. Röckner, and S. V. Shaposhnikov, On positive and probability solutions of the stationary Fokker-Planck-Kolmogorov equation, Dokl. Math. 85 (2012), 350-354.

[8] V. I. Bogachev, M. Röckner, and V. Stannat, Uniqueness of solutions of elliptic equations and uniqueness of invariant measures of diffusions. (Russian) Mat. Sb. 193 (2002), no. 7, 3-36; translation in Sb. Math. 193 (2002), no. 7-8, 945-976.

[9] R. Bowen, Equilibrium states and the ergodic theory of Anosov diffeomorphisms. Lecture Notes in Mathematics, Vol. 470. Springer-Verlag, Berlin-New York, 1975. i+108 pp.

[10] X. Chen, C. Caginalp, J. Hao, and Y. Zhang, Effects of white noise in multistavle dynamics, Discrete Contin. Dyn. Syst. Ser. B 18 no. 7 (2013), 1805-1825.

[11] W. Cowieson and L.-S. Young, SRB measures as zero-limits, Ergodic Theorey Dynam. Systems 25 (2005), $1115-1138$.

[12] R. L. Dobrushin, The description of a random field by means of conditional probabilities and conditions of its regularity, Theory Probab. Appl. 13 (1968), 197-224.

[13] R. L. Dobrushin, Gibbsian random fields for lattice systems with pairwise interactions, Funct. Anal. Appl. 2 (1968), 292-301.

[14] R. L. Dobrushin, The problem of uniqueness of a Gibbsian random field and the problem of phase transitions, Funct. Anal. Appl. 2 (1968), 302-312.

[15] H.-O. Georgii, Gibbs Measures and Phase Transitions, de Gruyter: Berlin, New York, 1988.

[16] W. Huang, M. Ji, Z. Liu, and Y. Yi, Integral identity and measure estimates for stationary Fokker-Planck equations, Ann. Probab. 43 (2015), 1712-1730.

[17] W. Huang, M. Ji, Z. Liu, and Y. Yi, Steady states of Fokker-Planck equations: I. Existence, J. Dyn. Diff. Equat. DOI 10.1007/s10884-015-9454-x

[18] W. Huang, M. Ji, Z. Liu, and Y. Yi, Steady states of Fokker-Planck equations: II. Non-existence, J. Dyn. Diff. Equat. DOI 10.1007/s10884-015-9470-x

[19] W. Huang, M. Ji, Z. Liu, and Y. Yi, Steady states of Fokker-Planck equations: III. Degenerate diffusion, J. Dyn. Diff. Equat., to appear.

[20] W. Huang, M. Ji, Z. Liu, and Y. Yi, Concentration and limit behaviors of stationary measures, submitted.

[21] C.-R. Hwang, Laplace's method revisited: weak convergence of probability measures, Ann. Probab. 8 (1980), 1177-1182.

[22] R. Jordan, D. Kinderlehrer, and F. Otto, The variational formulation of the Fokker-Planck euation, SIAM J. Math. Anal. 29 No. 1 (1998), 1-17.

[23] R. Khasminskii, Principle of averaging for parabolic and elliptic differential equations and for Markov processes with small diffusion, Theory Probab. Appl. 8 (1963), 1-21.

[24] Y. Kifer, On small random perturbations of some smooth dynamical systems, Izv. Akad. Nauk SSSR 38 (1974), 1083-1107. 
[25] O. E. Lanford, Entropy and equilibrium states in classical statistical mechanics. In "statistical mechanics and mathematical problems" (ed. A. Lenard), Lecture Notes in Physics 20, pp. 1-113. Springer, Berlin, 1973.

[26] O. E. Lanford, and D. Ruelle, Observables at infinity and states with short range correlations in statistical mechanics, Comm. Math. Phys. 13 (1969), 194-215.

[27] M. B. Nevel'son, The behavior of an invariant measure of a diffusion process with small diffusion on a circle, Theory Probab. Appl. 9 (1964), 125-130.

[28] A. D. Wentzell and M. Freidlin, On small random perturbations of dynamical systems, Russian Math. Surveys 25 (1970), 1-55.

[29] L.-S. Young, Stochastic stability of hyperbolic attractors, Ergodic Theorey Dynam. Systems 6 (1986), $311-319$.

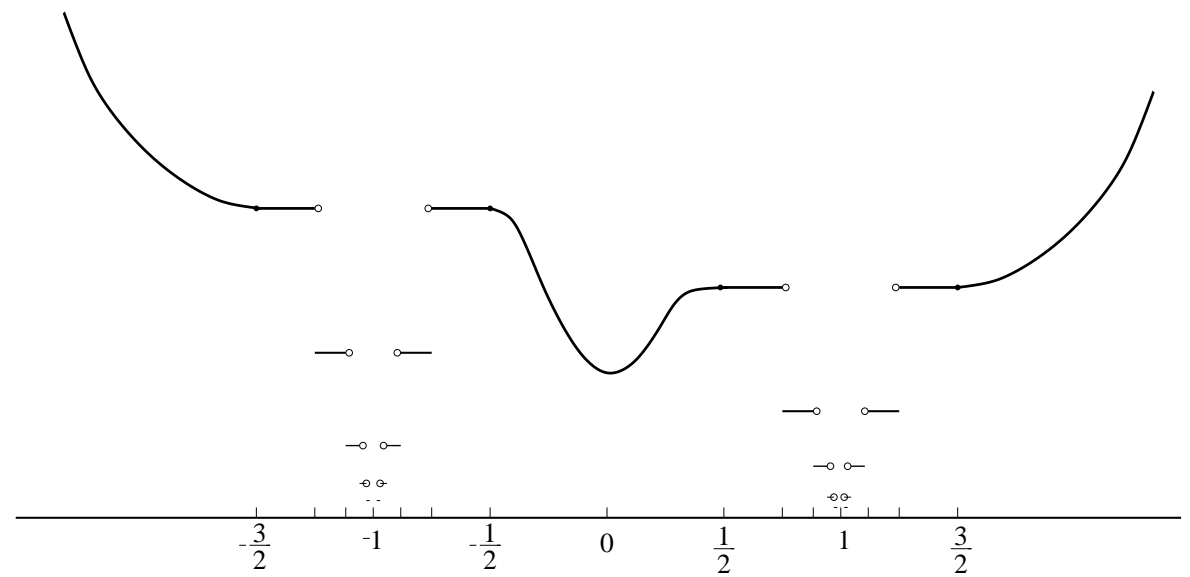

FiguRE 1. Graph of $f$

W. Huang: School of Mathematics, Sichuan University, Chengdu 610064, Sichuan, PRC and School of Mathematical Sciences, University of Science and Technology of China, Hefei 230026 , ANHui, PRC

E-mail address: wenh@mail.ustc.edu.cn

M. Ji: Academy of Mathematics and System Sciences, Chinese Academy of Sciences, Beijing 100080, PRC

E-mail address: jimin@math.ac.cn

Z. Liu: School of Mathematical Sciences, Dalian University of Technology, Dalian 116024, PRC

E-mail address: zxliu@dlut.edu.cn

Y. Yi: Department of Mathematical \& Statistical Sci, University of Alberta, Edmonton Alberta, Canada T6G 2G1, School of Mathematics, Jilin University, Changchun, 130012, PRC, and School of Mathematics, Georgia Institute of Technology, Atlanta, GA 30332, USA

E-mail address: yi@math.gatech.edu,yingfei@ualberta.ca 